\title{
Comment: should we diagnose MCI in Parkinson disease?
}

\author{
Amos D. Korczyn
}

Published online: 24 July 2011

(C) Springer-Verlag 2011

Both Rektorova and Martinez-Horta, and Kulisevsky agree that Parkinson disease (PD) patients have frequent cognitive impairment already at disease onset, which progresses at a varying rate, terminating in dementia in a substantial number of patients. Cognitive deterioration in PD follows an indolent course, like that seen in other neurodegenerative diseases, and it is an arbitrary decision to term any section of this process as mild cognitive impairment (MCI), or by any other name. It will be difficult to justify a "starting point" of MCI (since deterioration preceded it even if not fulfilling criteria), and even the conversion to dementia is more complicated to define than in Alzheimer disease (AD), since activities of daily living are impaired in PD by the motor disability, as well as by apathy, depression, etc. which precludes a clear-cut determination as it applies, for example, to AD.

The term MCI has been coined to denote the predementia cognitive impairment stage of $\mathrm{AD}$ and has attracted objections (Ash and Korczyn 2011). Borrowing it to other disorders should not be automatic. Several difficulties are stressed in both papers. In addition, it must be recalled that many of the PD patients have a unique premorbid personality, they frequently have depression, apathy and somnolence, are treated by drugs and of course have motor impairment - each of which could interfere with the execution and interpretation of neuropsychological tests.

The common definition of MCI depends on complaints by the patient (or a close informant). This is particularly true for memory impairment, and it is critical to employ

A. D. Korczyn ( $\square)$

The Sieratzki Chair of Neurology, Sackler School of Medicine, Tel-Aviv University, 69978 Ramat-Aviv, Israel

e-mail: amoskor@post.tau.ac.il

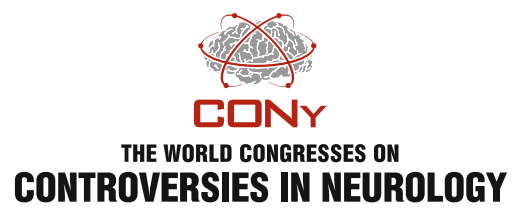

this parameter since a deterioration from a previous functional level is implied and there are usually no premorbid scores of cognitive testing in AD. However, accepting that in PD the first cognitive manifestation may be executive dysfunction, what should be the complaints? Clearly, in PD patients these cannot be just slowness! At present, this issue has not been addressed.

Both Rektorova and Martinez-Horta, and Kulisevsky emphasize the variability of cognitive deterioration. At present, there are few data on factors influencing the rate of decline. One of the important factors may be comorbidities, such as co-existent AD or vascular changes (Korczyn 2002). These, of course, will complicate further any interpretation of cognitive testing.

Another very important issue is why diagnose clinically inconspicuous or very mild cognitive impairment in PD? In $\mathrm{AD}$, the reason given is that disease modifying therapies are being developed which should be used early in the course of the disease. Since no such therapies are being developed against the cognitive deterioration in PD, the benefits from diagnosing an incipient dementia state are not clear. PD patients know that they face deterioration, particularly motor decline. They will not benefit from being told that they have been identified as being more likely than other PD patients to develop dementia as well.

\section{References}

Ash EL, Korczyn AD (2011) Is amnestic mild cognitive impairment a useful concept? Aging Health 7:107-122

Korczyn AD (2002) Mixed dementia-the most common cause of dementia. Ann NY Acad Sci 977:129-134 